\title{
Do the most frequently used dynamic panel data estimators have the best performance in a small sample? A Monte Carlo comparison
}

\author{
Blanka Škrabić Perić * \\ Faculty of Economics, Business and Tourism, University of Split \\ Cvite Fiskovića 5, 21000 Split, Croatia \\ E-mail: 〈bskrabic@efst.hr〉
}

\begin{abstract}
Differenced GMM and system GMM estimators are the two most frequently used dynamic panel estimators. Regardless the fact that both estimators are proposed for samples with a large $\mathrm{N}$ and short $\mathrm{T}$, both of them are frequently used for small samples. Therefore, this paper compares the small sample properties of these two estimators with standard dynamic LSDV and LSDV biascorrected estimators to examine the justification of their frequent use. Data set dimensions are formed considering dimensions of previous empirical studies that use dynamic panel data on small samples. The results show that LSDV bias-corrected estimator has the smallest RMSE in almost every design while in terms of bias, the results are mixed. LSDV bias-corrected outperforms both GMM estimators in terms of bias in design when the number of individuals is 10 and the number of time periods is 30. GMM estimators show somewhat better properties in terms of bias in design when the number of individuals is 30 and the number of time periods is 10 .
\end{abstract}

Keywords: dynamic panel data, GMM estimators, LSDV bias-corrected, small sample

Received: September 30, 2018; accepted: March 15, 2019; available online: July 4, 2019

DOI: 10.17535 /crorr.2019.0005

\section{Introduction}

Panel data analysis has become very popular in recent empirical studies. To estimate panel data, there are several dynamic panel data estimators with different properties. Most of the proposed estimators are suitable for estimation panel data with a large number of cross sections. However, empirical studies often deal with data set with a small number of cross sections and a small number of time periods. The properties of all estimators in small samples are poorly investigated. Therefore, Monte Carlo evidence is necessary to gauge which of these estimators are more acceptable for small data set.

The most popular dynamic panel data estimators are the difference GMM (AB) estimator proposed by Arellano and Bond [2] and the system GMM estimator (BB) proposed by Blundell and Bond [5]. They are used for small sample analysis regardless the fact that both estimators are proposed for a data set with a large number of cross sections and a small number of time periods. Additionally, Lillo and Torrecillas [22] warn of the limitations of GMM estimators in case when the number of individuals is small. Therefore, the question arises whether these estimators are suitable for the assessment of small samples. On the other hand, Least Squares Dummy Variables corrected estimator (LSDVc), proposed by Kiviet [21] for a balanced panel data and upgraded by Bruno [11] for unbalanced panel data, showed better properties in previous Monte Carlo simulations for a small sample [10, 13, 20]. However, it is rarely used in empirical analyses. Nonetheless, these studies are still not relevant to conclude that the LSDVc

${ }^{*}$ Corresponding author. 
estimator is superior for a small sample for several reasons. First, [13] and [20] did not consider properties of system GMM. Second, [20] ignored bias of the coefficient of independent variable and did not consider moderately persistent dependent and independent variables. Third, [10] considered the $\mathrm{BB}$ estimator, but he considered only data sets where the number of time series is bigger or the same as the number of cross sections.

Therefore, this research upgrades previous Monte Carlo studies in several ways. First, panel data dimensions are selected considering the characteristics of the existing economic empirical studies. Considering the number of cross sections and time dimension of existing empirical research, various dimensions of data set for Monte Carlo simulation are formed. It is known that the results of Monte Carlo simulation are more useful when simulated data set dimensions are close to real economic data sets [19]. Most of the existing simulation studies compared properties of estimators where the number of cross sections was at least 100.

Second, the properties of dynamic panel data estimators on small samples are rarely investigated. Namely, existing simulation studies compared properties of similar estimators. For example, they compared the properties of several GMM estimators or proposed some new estimators and compared its properties with previous estimators. This paper compares the properties of $\mathrm{AB}$ and $\mathrm{BB}$ as the most popular and frequently used in small sample analysis with standard LSDV and LSDVc estimators which are rarely used although they have shown better properties in previous Monte Carlo simulation studies.

In addition, this research gives a detail review of previous Monte Carlo studies which compared the properties of dynamic panel data on finite samples.

The rest of the paper is organized as follows. Section 2 gives literature review of previous Monte Carlo simulation studies with a focus on studies which deal with small samples. Section 3 describes Monte Carlo methodology. Section 4 presents and discusses Monte Carlo results. Section 5 gives conclusions and provides some general guidelines when choosing a dynamic panel data estimator for a small data set.

\section{Previous Monte Carlo simulations}

Most Monte Carlo studies are conducted to compare properties of a new estimator with existing estimators [2, 6, 16, 21, 23]. Additionally, it is known that GMM estimators have become very popular and are frequently used in empirical research. A large number of Monte Carlo simulation studies evaluate the properties of GMM estimators in various designs and compare the properties of several GMM estimators with others [4, 6, 7, 14, 15]. All of these studies deal with data set where the number of cross sections is at least 100 .

On the other hand, some empirical studies try to find the estimator which is the most appropriate one for some specific problem. Most studies are performed to evaluate the properties of estimators commonly used in the growth regression estimators [3, 9, 18, 19, 25].

Flannery and Hankins [17] simulated data sets that resemble "real" corporate finance data to compare the properties of seven dynamic panel data estimators. They found that LSDVc is the most accurate estimator in the absence of endogenous independent variables and second order serial correlation while BB and LSDV are the most accurate ones when those problems exist. Mertens [24] compared the properties of different panel estimators for firm growth model. In simulation studies he found that the BB estimator outperforms LSDV, $\mathrm{AB}$ and Anderson and Hsiao (AH) estimators [1] in all simulated designs. His simulated designs consist of a large number of individuals, i.e. 100 or 1000.

However, there is only limited research that tries to propose some guidelines for economic researchers. On the one hand, [8] compared the widely used econometric methods for dynamic panel data methods and provided a guide to micro data methods and practice. He focused his research on panels where large numbers of individuals are observed for a small number of time periods. 
On the other hand, the most popular research on small sample characteristics is provided by Judson and Owen [20]. They evaluated the properties of estimators for dynamic panel data with characteristics of many macroeconomic panel data sets. They tried to provide a guide for choosing an appropriate estimator for various dimensions of panel data set. In their research, the Monte Carlo study was performed to compare the properties of four estimators: $\mathrm{AH}$, one step and two step AB estimator, LSDVc, Ordinary Least Squares (OLS), and LSDV estimator. The number of cross sections took values 20 or 100 while time dimension takes on values 5 , 10, 20, and 30. They gave recommendations on how to choose the appropriate estimator with respect to the time dimension. For $T \leq 10$ they suggested the LSDVc estimator for balanced panels and the difference GMM for an unbalanced panels. For $T=20$, LSDVc was proposed for balanced panels while difference GMM or AH were proposed for unbalanced panels. For $T=30$, LSDVc showed the best properties for balanced panels while LSDV estimator was the best choice for unbalanced panels. However, in the existing empirical studies the number of cross sections is often less than 20 .

[13] considered properties of various dynamic panel data estimators when both dimension are really small. Precisely, they analyzed data sets with the following dimensions: $(N, T)=$ $\{(10,10),(10,20),(20,10)\}$ and they compared the properties of LSDV, AH, difference GMM (AB), and LSDVc estimator. The results show that LSDVc outperforms all estimators except $\mathrm{AH}$ in terms of bias while LSDVc outperforms all estimators in terms of RMSE.

Bruno [5] derived LSDVc for unbalanced data. He also compared finite properties of LSDVc estimator for unbalanced data with LSDV estimator, AB, AH, and BB estimators [4]. Furthermore, [4] included system GMM in research but he considered only data set with moderately and large time span $(N, T)=\{(20,20),(10,40)\}$. Also, all simulated data sets have smaller or the same number of cross sections as time periods and the superiority of LSDVc estimator is expected.

The results of existing studies $[11,13,20]$ are not relevant for a general decision for several reasons. [13] and [20] did not consider properties of the BB estimator. On the other hand, Bruno included the $\mathrm{BB}$ estimator but he considered data set with moderately and large time periods. Additionally, some data set dimensions which are usually used in empirical studies are not covered by the existing simulation studies. Therefore, researchers often use panel data with a small number of cross sections and time periods but there is no unique guide as to which estimator is the best one in their situation. As a consequence, we found that researchers use different estimators for similar data dimensions. To determine the most frequently used data set dimensions and used dynamic panel data estimators, we collected more than 20 papers from relevant economic journals which deal with small samples and use dynamic panel data (table with considered papers is omitted due to the limited number of pages but is available on request). Several data set dimensions for simulation were derived from these papers. Therefore, six different data set dimensions are considered: $(N, T)=\{(15,10),(10,15), \quad(12,12),(30,10), \quad(10,30), \quad(17,17)\}$. Additionally, from the literature reviewed it is additionally confirmed that $\mathrm{AB}$ and $\mathrm{BB}$ estimators are frequently used in these empirical studies.

\section{Simulation methodology}

Following previous empirical studies [10, 11, 13, 20, 21, 23], the dynamic fixed effect model is considered:

$$
y_{i t}=\gamma y_{i, t-1}+\beta x_{i t}+\alpha_{i}+\varepsilon_{i t}, \quad|\gamma|<1, \quad i=1,2, \ldots, N \quad t=1,2, \ldots, T,
$$

where $y_{i t}$ is the dependent variable, $y_{i, t-1}$ is the lagged dependent variable, $x_{i t}$ is the strictly exogenous explanatory variable, $\alpha_{i}$ is an unobserved individual effect and $\varepsilon_{i t}$ is an unobserved white noise disturbance. $\gamma$ and $\beta$ are regression coefficients. It is assumed that $\alpha_{i} \sim N\left(0, \sigma_{\alpha}^{2}\right)$, $\varepsilon_{i t} \sim N\left(0, \sigma_{\varepsilon}^{2}\right)$ and $E\left(x_{i t}, \varepsilon_{j s}\right)=0 \forall i, j, t, s$. 
Also, $x_{i t}$ is generated as an autoregressive process:

$$
x_{i t}=\rho x_{i, t-1}+\xi_{i t}, \quad i=1,2, \ldots, N \quad t=1,2, \ldots, T,
$$

where $x_{i, t-1}$ is the lagged $x_{i t}$ variable, $\rho$ is the autoregression coefficient and $\xi_{i t}$ is the unobserved white noise disturbance $\xi_{i t} \sim N\left(0, \sigma_{\xi}^{2}\right)$. In addition to $\beta$ and $\rho, \sigma_{\xi}^{2}$ also determines the relation between $y_{i t}$ and $x_{i t}$ and it has been calculated from other parameters of the model as follows:

$$
\sigma_{\xi}^{2}=\left[\sigma_{s}^{2}-\frac{\gamma^{2}}{1-\gamma^{2}} \sigma_{\varepsilon}^{2}\right]\left[1+\frac{(\gamma+\rho)^{2}}{1+\gamma \rho}(\gamma \rho-1)-(\gamma \rho)^{2}\right] \frac{1}{\beta^{2}},
$$

where

$$
\sigma_{s}^{2}=\operatorname{Var}\left(\nu_{i t}-\varepsilon_{i t}\right), \quad \nu_{i t}=y_{i t}-\frac{1}{1-\gamma} \alpha_{i}
$$

and it is called signal to noise ratio.

The higher $\sigma_{s}^{2}$ the more useful $x_{i t}$ is in explaining $y_{i t}$. In this research, $\sigma_{s}^{2}$ takes the value 2. Similar simulation studies take signal ratio 2 and 8 . In this paper, signal ratio 8 is omitted because of a lack of space (the results are available on request). Therefore, we considered only more difficult designs. The results in designs where signal ratio is 8 have smaller bias and RMSE. The values of coefficient $\gamma$ are $0.2,0.5$ and 0.8 because in macroeconomics and microeconomics research variables can be low, moderately or high persistent. Coefficient $\beta$ is calculated by $\beta=1-\gamma$ and a change in $\gamma$ affects only the short-run dynamics relationship between independent and dependent variables and not the steady-state relationship (like in most of the previously mentioned research). Therefore, coefficient $\beta$ also alternates between the values of $0.2,0.5$ and 0.8. $\sigma_{\varepsilon}^{2}$ is normalized to 1 while $\sigma_{\alpha}^{2}$ is calculated as $\sigma_{\alpha}^{2}=\sigma_{\varepsilon}^{2}(1-\gamma)$. In total, 18 different data sets are generated with 1000 replications (code xtrasim for Stata provided by [12] is used).

\section{Results and discussion}

In this section, the results of average absolute bias (avBias) and average RMSE (avRMSE) of $\gamma$ and $\beta$ for LSDV, AB, BB and LSDVc estimators are shown in Tables: 1-6.

From given results it is obvious that the LSDVc estimator has the smallest RMSE in comparison to other estimators in all simulated designs for all combinations of a number of cross sections, time periods, values of $\gamma, \beta$ and $\rho$. On the other hand, in terms of bias, LSDVc does not produce the best results in all designs and a detailed analysis is necessary.

In Table 1, a design with 15 cross sections and 10 time periods is considered. LSDVc produces the smallest bias for $\gamma=0.2$, while for moderately and high persistent BB shows the least bias (Table 1). For parameter $\beta$ the BB estimator produces the smallest bias for $\gamma=0.5$, while LSDVc produces the smallest bias when $\gamma=0.8$. For $\gamma=0.2$, the results are mixed. For $\rho=0.2 \mathrm{BB}$ produces the smallest bias of $\beta \mathrm{AB}$ produces the smallest bias while for $\rho=0.8$ LSDVc produces the smallest bias. From the results, it can be concluded that BB is superior in terms of $\gamma$ and $\beta$ when the dependent variable is moderately persistent.

If average absolute bias is considered, BB produces the smallest bias for $\gamma$, while LSDVc produces the smallest bias for $\beta$. It can also be concluded that $\mathrm{BB}$ outperforms $\mathrm{AB}$ in both coefficients.

In Table 2, a design with 12 cross sections and 12 time periods is considered. In Table 2, LSDVc produces the smallest bias for $\gamma=0.2$ while for moderately and high persistent BB it shows the least bias. For parameter $\beta$, LSDVc produces the smallest bias in 6 out of 9 designs. Precisely, in all designs when $\gamma=0.2$, in designs when qgamma $=0.5$ and $\rho=0.8$ and $\gamma=0.8$ and $\rho=0.2,0.5$. 
Do the most frequently used dynamic panel data estimators have the best performance in a small sample? 49

\begin{tabular}{|c|c|c|c|c|c|c|c|c|c|c|}
\hline$\gamma$ & $\beta$ & $\rho$ & $L S D V \gamma$ & $L S D V \beta$ & $A B \gamma$ & $A B \beta$ & $B B \gamma$ & $B B \beta$ & $L S D V_{C} \gamma$ & $L S D V_{C} \beta$ \\
\hline \multirow[t]{6}{*}{0.2} & 0.8 & 0.2 & -0.0523 & 0.0053 & -0.0130 & 0.0002 & 0.0163 & 0.0001 & -0.0035 & 0.0040 \\
\hline & & & 0.0745 & 0.0562 & 0.1035 & 0.0774 & 0.0935 & 0.0744 & 0.0556 & 0.0561 \\
\hline & & 0.5 & -0.0636 & 0.0189 & -0.0216 & 0.0026 & 0.0187 & -0.0059 & -0.0071 & 0.0052 \\
\hline & & & 0.0850 & 0.0686 & 0.1223 & 0.0954 & 0.1061 & 0.0919 & 0.0598 & 0.0662 \\
\hline & & 0.8 & -0.0922 & 0.0457 & -0.0376 & 0.0120 & 0.0280 & -0.0153 & -0.0131 & 0.0085 \\
\hline & & & 0.1141 & 0.1066 & 0.1532 & 0.1531 & 0.1301 & 0.1472 & 0.0733 & 0.0961 \\
\hline \multirow[t]{6}{*}{0.5} & 0.5 & 0.2 & -0.0910 & 0.0012 & -0.0328 & -0.0017 & 0.0076 & -0.0003 & -0.0115 & 0.0025 \\
\hline & & & 0.1078 & 0.0459 & 0.1404 & 0.0628 & 0.1085 & 0.0599 & 0.0631 & 0.0460 \\
\hline & & 0.5 & -0.1062 & 0.0163 & -0.0491 & 0.0021 & 0.0050 & -0.0014 & -0.0179 & 0.0046 \\
\hline & & & 0.1226 & 0.0614 & 0.1640 & 0.0842 & 0.1173 & 0.0803 & 0.0683 & 0.0591 \\
\hline & & 0.8 & -0.1400 & 0.0472 & -0.0718 & 0.0157 & 0.0039 & -0.0027 & -0.0281 & 0.0101 \\
\hline & & & 0.1568 & 0.1069 & 0.1992 & 0.1503 & 0.1358 & 0.1429 & 0.0825 & 0.0944 \\
\hline \multirow[t]{8}{*}{0.8} & 0.2 & 0.2 & -0.2381 & -0.0062 & -0.1201 & -0.0022 & -0.0229 & 0.0031 & -0.0771 & -0.0018 \\
\hline & & & 0.2505 & 0.0772 & 0.2557 & 0.1040 & 0.1365 & 0.1004 & 0.1181 & 0.0778 \\
\hline & & 0.5 & -0.2433 & 0.0052 & -0.1283 & 0.0054 & -0.0252 & 0.0069 & -0.0820 & 0.0009 \\
\hline & & & 0.2557 & 0.1139 & 0.2617 & 0.1604 & 0.1382 & 0.1533 & 0.1219 & 0.1115 \\
\hline & & 0.8 & -0.2508 & 0.0272 & -0.1344 & 0.0227 & -0.0280 & 0.0170 & -0.0877 & 0.0058 \\
\hline & & & 0.2632 & 0.2185 & 0.2674 & 0.3358 & 0.1408 & 0.3024 & 0.1268 & 0.2048 \\
\hline & & Bias & 0.1184 & 0.0193 & 0.0567 & 0.0072 & 0.0173 & 0.0055 & 0.0325 & 0.0048 \\
\hline & avR & ISE & 0.1589 & 0.0950 & 0.1853 & 0.1359 & 0.1230 & 0.1281 & 0.0855 & 0.0902 \\
\hline
\end{tabular}

Table 1: $\gamma$ and $\beta$ bias and RMSE using various estimators when $N=15, T=10$ and $\sigma_{s}^{2}=2$

\begin{tabular}{|rcc|rrrrrrrr|}
\hline$\gamma$ & $\beta$ & $\rho$ & $L S D V \gamma$ & $L S D V \beta$ & $A B \gamma$ & $A B \beta$ & $B B \gamma$ & $B B \beta$ & $L S D V_{C} \gamma$ & $L S D V_{C} \beta$ \\
\hline 0.2 & 0.8 & 0.2 & -0.0407 & 0.0020 & -0.0152 & -0.0039 & 0.0162 & -0.0078 & -0.0013 & -0.0003 \\
& & & 0.0690 & 0.0577 & 0.1002 & 0.0748 & 0.1009 & 0.0787 & 0.0576 & 0.0576 \\
& & 0.5 & -0.0407 & 0.0020 & -0.0152 & -0.0039 & 0.0162 & -0.0078 & -0.0013 & -0.0003 \\
& & & 0.0690 & 0.0577 & 0.1002 & 0.0748 & 0.1009 & 0.0787 & 0.0576 & 0.0576 \\
& & & -0.0732 & 0.0387 & -0.0333 & 0.0063 & 0.0293 & -0.0263 & -0.0093 & 0.0052 \\
& & 0.0995 & 0.1030 & 0.1423 & 0.1518 & 0.1432 & 0.1560 & 0.0720 & 0.0950 \\
\hline 0.5 & 0.5 & 0.2 & -0.0720 & 0.0000 & -0.0305 & -0.0037 & 0.0061 & -0.0052 & -0.0080 & -0.0006 \\
& & & 0.0935 & 0.0472 & 0.1305 & 0.0618 & 0.1184 & 0.0630 & 0.0634 & 0.0471 \\
& 0.5 & -0.0841 & 0.0137 & -0.0423 & -0.0005 & 0.0027 & -0.0072 & -0.0131 & 0.0017 \\
& & 0.1044 & 0.0619 & 0.1497 & 0.0827 & 0.1287 & 0.0847 & 0.0667 & 0.0601 \\
& 0.8 & -0.1130 & 0.0429 & -0.0651 & 0.0097 & 0.0016 & -0.0134 & -0.0226 & 0.0081 \\
& & 0.1909 & 0.0867 & 0.1757 & 0.0839 & 0.1287 & 0.0868 & 0.0739 & 0.0611 \\
\hline 0.8 & 0.2 & 0.2 & -0.1950 & -0.0069 & -0.1145 & -0.0081 & -0.0413 & -0.0076 & -0.0617 & -0.0054 \\
& & 0.2092 & 0.0795 & 0.2265 & 0.1028 & 0.1743 & 0.1057 & 0.1051 & 0.0796 \\
& 0.5 & -0.2000 & 0.0045 & -0.1211 & -0.0045 & -0.0422 & -0.0075 & -0.0665 & -0.0025 \\
& & 0.2141 & 0.1165 & 0.2308 & 0.1576 & 0.1728 & 0.1610 & 0.1085 & 0.1132 \\
& & & -0.2081 & 0.0279 & -0.1281 & 0.0040 & -0.0446 & -0.0032 & -0.0732 & 0.0047 \\
& & 0.2219 & 0.2196 & 0.2350 & 0.3281 & 0.1690 & 0.3642 & 0.1136 & 0.2034 \\
\hline & & avBias & 0.0954 & 0.0154 & 0.0534 & 0.0049 & 0.0222 & 0.0080 & 0.0256 & 0.0032 \\
& avRMSE & 0.1413 & 0.0922 & 0.1657 & 0.1243 & 0.1374 & 0.1310 & 0.0798 & 0.0861 \\
\hline
\end{tabular}

Table 2: $\gamma$ and $\beta$ bias and RMSE using various estimators when $N=T=12$ and $\sigma_{s}^{2}=2$ 


\begin{tabular}{|rcc|rrrrrrrr|}
\hline$\gamma$ & $\beta$ & $\rho$ & $L S D V \gamma$ & $L S D V \beta$ & $A B \gamma$ & $A B \beta$ & $B B \gamma$ & $B B \beta$ & $L S D V_{C} \gamma$ & $L S D V_{C} \beta$ \\
\hline 0.2 & 0.8 & 0.2 & -0.0354 & 0.0057 & -0.0316 & -0.0035 & 0.0167 & -0.0023 & -0.0048 & 0.0033 \\
& & & 0.0610 & 0.0530 & 0.1200 & 0.0768 & 0.1128 & 0.0783 & 0.0510 & 0.0528 \\
& & 0.5 & -0.0430 & 0.0152 & -0.0424 & 0.0027 & 0.0132 & -0.0059 & -0.0077 & 0.0040 \\
& & & 0.0585 & 0.0529 & 0.1132 & 0.0734 & 0.1079 & 0.0763 & 0.0489 & 0.0501 \\
& & 0.8 & -0.0613 & 0.0344 & -0.0776 & 0.0153 & 0.0039 & -0.0146 & -0.0116 & 0.0061 \\
& & & 0.0904 & 0.0930 & 0.1926 & 0.1681 & 0.1587 & 0.1602 & 0.0701 & 0.0864 \\
\hline 0.5 & 0.5 & 0.2 & -0.0595 & 0.0038 & -0.0665 & -0.0067 & -0.0123 & -0.0022 & -0.0100 & 0.0025 \\
& & & 0.0805 & 0.0431 & 0.1723 & 0.0661 & 0.1307 & 0.0620 & 0.0569 & 0.0429 \\
& 0.5 & -0.0692 & 0.0149 & -0.0991 & -0.0030 & -0.0281 & -0.0033 & -0.0142 & 0.0040 \\
& & 0.0911 & 0.0560 & 0.2115 & 0.0850 & 0.1503 & 0.0828 & 0.0631 & 0.0539 \\
& 0.8 & -0.0904 & 0.0381 & -0.1351 & 0.0125 & -0.0422 & -0.0018 & -0.0199 & 0.0076 \\
& & 0.1135 & 0.0920 & 0.2551 & 0.1487 & 0.1895 & 0.1563 & 0.0748 & 0.0825 \\
\hline 0.8 & 0.2 & 0.2 & -0.1546 & 0.0006 & -0.2156 & -0.0105 & -0.0936 & -0.0029 & -0.0458 & 0.0013 \\
& & 0.1707 & 0.0724 & 0.3362 & 0.0983 & 0.2153 & 0.0993 & 0.0915 & 0.0723 \\
& 0.5 & -0.1582 & 0.0092 & -0.2232 & 0.0002 & -0.1005 & 0.0035 & -0.0490 & 0.0022 \\
& & 0.1745 & 0.1035 & 0.3403 & 0.1554 & 0.2228 & 0.1535 & 0.0942 & 0.0999 \\
& & & -0.1639 & 0.0248 & -0.2285 & 0.0447 & -0.1068 & 0.0648 & -0.0532 & 0.0029 \\
& & 0.1802 & 0.1922 & 0.3566 & 0.5086 & 0.2418 & 0.5659 & 0.0976 & 0.1772 \\
\hline & & avBias & 0.0775 & 0.0163 & 0.1024 & 0.0103 & 0.0401 & 0.0105 & 0.0209 & 0.0038 \\
& avRMSE & 0.1136 & 0.0807 & 0.2272 & 0.1457 & 0.1600 & 0.1519 & 0.0678 & 0.0748 \\
\hline
\end{tabular}

Table 3: $\gamma$ and $\beta$ bias and RMSE using various estimators when $N=10, T=15$ and $\sigma_{s}^{2}=2$

\begin{tabular}{|rcc|rrrrrrrr|}
\hline$\gamma$ & $\beta$ & $\rho$ & $L S D V \gamma$ & $L S D V \beta$ & $A B \gamma$ & $A B \beta$ & $B B \gamma$ & $B B \beta$ & $L S D V_{C} \gamma$ & $L S D V_{C} \beta$ \\
\hline 0.2 & 0.8 & 0.2 & -0.0500 & 0.0042 & -0.0056 & -0.0004 & 0.0133 & -0.0050 & -0.0010 & 0.0028 \\
& & & 0.0627 & 0.0427 & 0.0702 & 0.0552 & 0.0680 & 0.0562 & 0.0400 & 0.0426 \\
& & 0.5 & -0.0600 & 0.0179 & -0.0107 & 0.0013 & 0.0168 & -0.0095 & -0.0034 & 0.0038 \\
& & & 0.0727 & 0.0535 & 0.0833 & 0.0690 & 0.0802 & 0.0704 & 0.0433 & 0.0508 \\
& & 0.8 & -0.0880 & 0.0456 & -0.0195 & 0.0048 & 0.0231 & -0.0196 & -0.0073 & 0.0069 \\
& & & 0.1008 & 0.0861 & 0.1083 & 0.1125 & 0.0996 & 0.1139 & 0.0539 & 0.0734 \\
\hline 0.5 & 0.5 & 0.2 & -0.0870 & 0.0005 & -0.0189 & -0.0013 & 0.0082 & -0.0038 & -0.0068 & 0.0018 \\
& & & 0.0964 & 0.0347 & 0.0943 & 0.0448 & 0.0829 & 0.0450 & 0.0455 & 0.0348 \\
& 0.5 & -0.1010 & 0.0156 & -0.0293 & 0.0005 & 0.0085 & -0.0064 & -0.0118 & 0.0036 \\
& & 0.1105 & 0.0475 & 0.1120 & 0.0608 & 0.0942 & 0.0612 & 0.0495 & 0.0449 \\
& 0.8 & -0.1340 & 0.0477 & -0.0412 & 0.0059 & 0.0105 & -0.0119 & -0.0191 & 0.0086 \\
& & 0.1440 & 0.0863 & 0.1395 & 0.1103 & 0.1090 & 0.1096 & 0.0608 & 0.0713 \\
\hline 0.8 & 0.2 & 0.2 & -0.2300 & -0.0069 & -0.0702 & -0.0023 & -0.0146 & -0.0022 & -0.0594 & -0.0015 \\
& & 0.2370 & 0.0577 & 0.1713 & 0.0744 & 0.1147 & 0.0748 & 0.0886 & 0.0580 \\
& 0.5 & -0.2350 & 0.0059 & -0.0755 & 0.0015 & -0.0168 & -0.0002 & -0.0633 & 0.0014 \\
& & 0.2416 & 0.0854 & 0.1767 & 0.1169 & 0.1165 & 0.1165 & 0.0920 & 0.0835 \\
& & & -0.2420 & 0.0335 & -0.0789 & 0.0119 & -0.0185 & 0.0066 & -0.0682 & 0.0089 \\
& & 0.2491 & 0.1651 & 0.1816 & 0.2487 & 0.1190 & 0.2341 & 0.0966 & 0.1531 \\
\hline & & avBias & 0.1139 & 0.0198 & 0.0324 & 0.0033 & 0.0145 & 0.0058 & 0.0241 & 0.0044 \\
& avRMSE & 0.1634 & 0.0838 & 0.1511 & 0.1258 & 0.1141 & 0.1296 & 0.0782 & 0.0838 \\
\hline
\end{tabular}

Table 4: $\gamma$ and $\beta$ bias and RMSE using various estimators when $N=30, T=10$ and $\sigma_{s}^{2}=2$ 
Do the most frequently used dynamic panel data estimators have the best performance in a small sample? 51

\begin{tabular}{|rcc|rrrrrrrr|}
\hline$\gamma$ & $\beta$ & $\rho$ & $L S D V \gamma$ & $L S D V \beta$ & $A B \gamma$ & $A B \beta$ & $B B \gamma$ & $B B \beta$ & $L S D V_{C} \gamma$ & $L S D V_{C} \beta$ \\
\hline 0.2 & 0.8 & 0.2 & -0.0290 & 0.0025 & -0.0104 & -0.0001 & 0.0159 & -0.0023 & -0.0023 & 0.0001 \\
& & & 0.0464 & 0.0385 & 0.0630 & 0.0508 & 0.0671 & 0.0524 & 0.0372 & 0.0385 \\
& & 0.5 & -0.0344 & 0.0107 & -0.0159 & 0.0024 & 0.0187 & -0.0070 & -0.0036 & 0.0004 \\
& & & 0.0524 & 0.0456 & 0.0739 & 0.0634 & 0.0763 & 0.0668 & 0.0408 & 0.0446 \\
& & 0.8 & -0.0493 & 0.0273 & -0.0263 & 0.0106 & 0.0251 & -0.0179 & -0.0056 & 0.0011 \\
& & & 0.0685 & 0.0670 & 0.0940 & 0.1010 & 0.0966 & 0.1047 & 0.0497 & 0.0614 \\
\hline 0.5 & 0.5 & 0.2 & -0.0492 & 0.0015 & -0.0221 & -0.0007 & 0.0030 & -0.0021 & -0.0059 & -0.0001 \\
& & & 0.0626 & 0.0313 & 0.0822 & 0.0412 & 0.0782 & 0.0423 & 0.0407 & 0.0312 \\
& 0.5 & -0.0564 & 0.0111 & -0.0323 & 0.0023 & 0.0040 & -0.0032 & -0.0084 & 0.0007 \\
& & 0.0700 & 0.0405 & 0.0957 & 0.0560 & 0.0869 & 0.0583 & 0.0439 & 0.0390 \\
& 0.8 & -0.0735 & 0.0313 & -0.0464 & 0.0117 & 0.0083 & -0.0052 & -0.0116 & 0.0024 \\
& & 0.0877 & 0.0667 & 0.1162 & 0.0987 & 0.1009 & 0.0995 & 0.0514 & 0.0584 \\
\hline 0.8 & 0.2 & 0.2 & -0.1298 & -0.0028 & -0.0723 & -0.0012 & -0.0287 & -0.0011 & -0.0313 & -0.0028 \\
& & 0.1385 & 0.0525 & 0.1411 & 0.0681 & 0.1106 & 0.0700 & 0.0617 & 0.0527 \\
& 0.5 & -0.1321 & 0.0049 & -0.0767 & 0.0042 & -0.0293 & 0.0018 & -0.0329 & -0.0029 \\
& & 0.1407 & 0.0733 & 0.1450 & 0.1054 & 0.1108 & 0.1063 & 0.0629 & 0.0717 \\
& & & -0.1361 & 0.0205 & -0.0802 & 0.0163 & -0.0303 & 0.0097 & -0.0354 & -0.0037 \\
& & 0.1448 & 0.1321 & 0.1490 & 0.2187 & 0.1118 & 0.2147 & 0.0648 & 0.1228 \\
\hline & & avBias & 0.0641 & 0.0125 & 0.0353 & 0.0055 & 0.0181 & 0.0049 & 0.0134 & 0.0016 \\
& avRMSE & 0.0902 & 0.0608 & 0.1067 & 0.0893 & 0.0932 & 0.0906 & 0.0504 & 0.0578 \\
\hline
\end{tabular}

Table 5: $\gamma$ and $\beta$ bias and RMSE using various estimators when $N=T=17$ and $\sigma_{s}^{2}=2$

\begin{tabular}{|ccc|rrrrrrrr|}
\hline$\gamma$ & $\beta$ & $\rho$ & $L S D V \gamma$ & $L S D V \beta$ & $A B \gamma$ & $A B \beta$ & $B B \gamma$ & $B B \beta$ & $L S D V_{C} \gamma$ & $L S D V_{C} \beta$ \\
\hline 0.2 & 0.8 & 0.2 & -0.0152 & 0.0036 & -0.0375 & -0.0081 & 0.0052 & -0.0033 & -0.0004 & 0.0018 \\
& & & 0.0389 & 0.0368 & 0.1303 & 0.0626 & 0.1103 & 0.0596 & 0.0362 & 0.0367 \\
& & 0.5 & -0.0184 & 0.0088 & -0.0520 & -0.0022 & -0.0066 & -0.0082 & -0.0013 & 0.0023 \\
& & & 0.0434 & 0.0431 & 0.1520 & 0.0701 & 0.1326 & 0.0682 & 0.0398 & 0.0424 \\
& & & -0.0268 & 0.0204 & -0.1155 & 0.0054 & -0.0334 & -0.0092 & -0.0026 & 0.0041 \\
& & 0.0539 & 0.0591 & 0.2236 & 0.1019 & 0.1861 & 0.1148 & 0.0478 & 0.0558 \\
\hline 0.5 & 0.5 & 0.2 & -0.0258 & 0.0031 & -0.0859 & -0.0135 & -0.0335 & -0.0070 & -0.0020 & 0.0016 \\
& & & 0.0452 & 0.0298 & 0.2065 & 0.0572 & 0.1497 & 0.0488 & 0.0378 & 0.0297 \\
& 0.5 & -0.0298 & 0.0094 & -0.1283 & -0.0110 & -0.0607 & -0.0080 & -0.0036 & 0.0025 \\
& & 0.0497 & 0.0382 & 0.2481 & 0.0637 & 0.1919 & 0.0608 & 0.0406 & 0.0371 \\
& 0.8 & -0.0397 & 0.0242 & -0.1974 & 0.0062 & -0.1004 & 0.0001 & -0.0057 & 0.0054 \\
& & 0.0600 & 0.0581 & 0.3249 & 0.1140 & 0.2517 & 0.1168 & 0.0464 & 0.0528 \\
\hline 0.8 & 0.2 & 0.2 & -0.0697 & 0.0027 & -0.3119 & -0.0233 & -0.2003 & -0.0139 & -0.0120 & 0.0019 \\
& & 0.0816 & 0.0500 & 0.4450 & 0.0719 & 0.3389 & 0.0703 & 0.0463 & 0.0498 \\
& 0.5 & -0.0713 & 0.0095 & -0.3244 & -0.0151 & -0.2096 & -0.0077 & -0.0132 & 0.0033 \\
& & 0.0832 & 0.0693 & 0.4565 & 0.1035 & 0.3593 & 0.1046 & 0.0472 & 0.0670 \\
& & & -0.0746 & 0.0267 & -0.3185 & 0.0546 & -0.2191 & 0.0771 & -0.0156 & 0.0083 \\
& & 0.0865 & 0.1165 & 0.4614 & 0.5841 & 0.3536 & 0.6522 & 0.0486 & 0.1065 \\
\hline & & avBias & 0.0346 & 0.0120 & 0.1461 & 0.0130 & 0.0830 & 0.0132 & 0.0055 & 0.0035 \\
& avRMSE & 0.0603 & 0.0556 & 0.2943 & 0.1366 & 0.2305 & 0.1440 & 0.0434 & 0.0531 \\
\hline
\end{tabular}

Table 6: $\gamma$ and $\beta$ bias and RMSE using various estimators when $N=10, T=30$ and $\sigma_{s}^{2}=2$ 
In Table 3, a design with 10 cross sections and 15 time periods is considered. From Table 3 , it can be concluded that LSDVc outperforms all other estimators in terms of bias of $\gamma$ regardless of the value of $\gamma$ and $\rho$. The results for bias of $\beta$ are mixed but LSDVc produces the smallest absolute average bias for a $\beta$. It is an interesting finding that LSDV outperforms the $\mathrm{AB}$ estimator in the sense of bias of parameter $\gamma$. In Table 4, a design with 30 cross sections and 10 time periods is considered. In this table, LSDVc produces the smallest bias for $\gamma$ when $\gamma=0.2$, while for moderately and highly persistent BB shows the least bias. For $\beta, \mathrm{AB}$ estimator produces the smallest bias in more than half of designs. A design with 17 cross sections and 17 time periods is considered (Table 5). LSDVc produces the smallest bias for $\gamma$ when $\gamma=0.2$, while for moderately and high persistent BB shows the least bias. For parameter $\beta$, LSDVc produces the smallest bias in 6 out of 9 designs. AB produces the smallest bias when $\gamma=\rho=0.2$, while BB produces the least bias for $\gamma=0.8$ and $r h o=0.2,0.5$. In Table 6, a design with 10 cross sections and 30 time periods is considered. In Table 6, LSDVc produces the smallest bias in almost all designs regardless of the value of parameters $\gamma$ and $\rho$. In terms of bias, LSDV outperforms $\mathrm{AB}$ and $\mathrm{BB}$ estimators in bias of $\gamma$. Additionally, LSDV outperforms the $\mathrm{AB}$ estimator in bias of $\beta$ as in [20], while $\mathrm{BB}$ outperforms LSDV in 6 out of 9 designs. From these results, it is obvious that LSDVc outperforms all other estimators in this design. The properties of sophisticated and very popular $\mathrm{BB}$ and $\mathrm{AB}$ can be compared with the LSDV estimator. Therefore, using some of GMM estimators instead of LSDVc is not correct. From a detailed analysis of all 6 tables, it can be concluded that GMM estimators do not have better properties than the LSDVc estimator in small samples. From Table 6, it is obvious that in designs when $(N, T)=(10,30)$ LSDVc outperforms both estimators. Additionally, in both designs where the number of time periods exceeds the number of cross sections $(N, T)=\{(10,30), \quad(10,30)\}$ LSDV has a smaller bias than the AB estimator for a $\gamma$ coefficient. This result is in line with [20] that the LSDV estimator outperforms AB when $T=20$.

The $\mathrm{BB}$ estimator outperforms the $\mathrm{AB}$ estimator in terms of bias of $\gamma$ in all designs. However, for two combinations of cross sections and time periods when sections $(N, T)=$ $\{(12,12), \quad(30,10)\} \mathrm{AB}$ outperforms $\mathrm{BB}$ in bias of $\beta$ in more than half of designs and in the average absolute bias of $\beta$. For quadratic design $(N, T)=\{(12,12),(17,17)\}$, the LSDVc estimator shows somewhat better properties than $\mathrm{BB}$ and $\mathrm{AB}$ estimators in terms of the average absolute bias of both parameters. For design $(N, T)=\{(15,10),(10,15)\}$ some general recommendations cannot be adopted. But if researchers know the characteristics of their data set (for example persistency of dependent or independent variables) and if they are interested in the precision of lagged dependent or independent variables, the results of this research can help them to choose the most appropriate dynamic panel data estimator for their research. For example, in design $(N, T)=(10,15) \mathrm{BB}$ shows the smallest bias for $\beta$ and $\gamma$ in all designs when $\gamma$ is moderately persistent.

\section{Conclusion and recommendations}

Simulation results did not give an estimator with the best performance for each data set dimensions but some conclusions can be adopted. From the results of this simulation study, it is obvious that the most popular and frequently used estimators difference GMM and system GMM do not show superiority in any simulated design. If RMSE is chosen as an indicator of the best estimator then LSDVc is the best choice for all designs. RMSE of AB and BB is comparable with bias LSDV estimator in almost all designs.

If the bias of estimator is considered as a criterion when choosing the best estimator, the results are somewhat mixed. Definitely the main finding of this research is that for the designs where the number of cross sections is 10 and the number of time periods is 30 , the LSDVc estimator shows smaller bias than $\mathrm{AB}$ and $\mathrm{BB}$ estimators. Further, the bias of $\mathrm{BB}$ and $\mathrm{AB}$ 
estimators is comparable with standard LSDV estimator. These results additionally confirm that researchers have to avoid using any of the two GMM estimators in data set with these characteristics regardless of their popularity and existing empirical studies which use one of the two estimators. For both quadratic designs, the LSDVc estimator shows somewhat better properties than $\mathrm{BB}$ and $\mathrm{AB}$ estimators in terms of average absolute bias in estimating independent variable.

For designs with 30 cross sections and 10 time periods, LSDVc is not the least biased. BB has somewhat smaller bias for lagged dependent variable while $\mathrm{AB}$ produces somewhat smaller bias in terms of independent variable.

For other designs, some general recommendations cannot be adopted. But if researchers know the characteristics of their data set and if they are interested in the precision of lagged dependent or independent variables, the results of this research can help them to choose the most appropriate dynamic panel data estimator for their research.

Finally, it can be concluded that this research gives some guidelines for using dynamic panel data but at the same time opens up numerous questions. This research does not evaluate all possible characteristics of data set as potential endogenous regressors, nonstationarity, ratio of variance of error components or some other characteristics. Namely, endogenous regressors require the use of additional instrumental variables so introducing them into regression makes sense only in designs where the number of cross sections is larger than time periods. Thus, a further research will be limited to the subset of simulated designs with endogenous regressors, where the number of cross sections is larger than time periods.

\section{References}

[1] Anderson, T. W. and Hsiao, C. (1982). Formulation and estimation of dynamic models using panel data. Journal of Econometrics, 18(1), 47-82.doi: 10.1016/0304-4076(82)90095-1

[2] Arellano, M. and Bond, S. (1991). Some Tests of Specification for Panel Data: Monte Carlo Evidence and an Application to Employment Equations. The Review of Economic Studies, 58(2), 277-297. doi: $10.2307 / 2297968$

[3] Bazzi, S. and Clemens, M. A. (2013). Blunt Instruments: Avoiding Common Pitfalls in Identifying the Causes of Economic Growth. American Economic Journal: Macroeconomics, 5(2), 152-186. doi: $10.1257 / \operatorname{mac} .5 \cdot 2.152$

[4] Behr, A. (2003). A comparison of dynamic panel data estimators: Monte Carlo evidence and an application to the investment function. Discussion Paper Series 1: Economic Studies, Deutsche Bundesbank Research Centre, 5. https://ideas.repec.org/p/zbw/bubdp1/4200.html

[5] Blundell, R. and Bond, S. (1998). Initial conditions and moment restrictions in dynamic panel data models. Journal of Econometrics, 87(1), 115-143. doi: 10.1016/s0304-4076(98)00009-8

[6] Blundell, R. and Bond, S. (2000). GMM Estimation with persistent panel data: an application to production functions. Econometric Reviews, 19(3), 321-340. doi: 10.1080/07474930008800475

[7] Blundell, R., Bond, S. and Windmeijer, F. (2001). Estimation in dynamic panel data models: Improving on the performance of the standard GMM estimator, 53-91. In Baltagi, B. H., Fomby, T. B. and Hill, R. C. (Eds): Nonstationary Panels, Panel Cointegration, and Dynamic Panels (Advances in Econometrics, Volume 15), Emerald Group Publishing Limited. doi: 10.1016/s07319053(00)15003-0

[8] Bond, S. R. (2002). Dynamic panel data models: a guide to micro data methods and practice. Portuguese Economic Journal, 1(2), 141-162. doi: 10.1007/s10258-002-0009-9

[9] Bond, S. R., Hoeffler, A. and Temple, J. R. W. (2001). GMM Estimation of Empirical Growth Models. Economics Papers, Economics Group, Nuffield College, University of Oxford, 21.

[10] Bruno, G. S. (2005a). Estimation and inference in dynamic unbalanced panel-data models with a small number of individuals. The Stata Journal: Promoting communicationss on statistics and Stata, 5(4), 473-500. doi: 10.1177/1536867x0500500401

[11] Bruno, G. S. (2005b). Approximating the bias of the LSDV estimator for dynamic unbalanced panel data models. Economics Letters, 87(3), 361-366. doi: 10.1016/j.econlet.2005.01.005 
[12] Bruno, G. S. (2005c). XTARSIM: Stata module to perform Monte Carlo analysis for dynamic panel data models. Statistical Software Components, Boston College Department of Economics, S453801. https://ideas.repec.org/c/boc/bocode/s453801.html

[13] Bun, M. J. and Kiviet, J. F. (2001). The accuracy of inference in small samples of dynamic panel data models. Tinbergen Institute Discussion Papers, Tinbergen Institute, 06/4. https://ideas . repec.org/p/tin/wpaper/20010006.html

[14] Bun, M. J. and Sarafidis, V. (2013). Dynamic panel data models. Econometrics Working Papers, University of Amstredam, 13/01. https://ideas.repec.org/p/ame/wpaper/1301.html

[15] Bun, M. J. and Windmeijer, F. (2010). The weak instrument problem of the system GMM estimator in dynamic panel data models. Econometrics Journal, 13(1), 95-126. doi: 10.1111/j.1368423X.2009.00299.x

[16] Everaert, G. (2013). Orthogonal to backward mean transformation for dynamic panel data models. The Econometrics Journal, 16(2), 179-221. doi: 10.1111/ectj.12001

[17] Flannery, M. J. and Hankins, K. W. (2013). Estimating dynamic panel models in corporate finance. Journal of Corporate Finance, 19, 1-19. doi: 10.1016/j.jcorpfin.2012.09.004

[18] Hauk, W. R. and Wacziarg, R. (2009). A Monte Carlo study of growth regressions. Journal of Economic Growth, 14(2), 103-147. doi: 10.1007/s10887-009-9040-3

[19] Islam, N. (2001). Small sample performance of dynamic panel data estimators in estimating the growth-convergence equation: A Monte Carlo study, 317-339. In Baltagi, B. H., Fomby, T. B. and Hill, R. C. (Eds): Nonstationary Panels, Panel Cointegration, and Dynamic Panels (Advances in Econometrics, Volume 15), Emerald Group Publishing Limited. doi: 10.1016/s07319053(00)15012-1

[20] Judson, R. A. and Owen, A. L. (1999). Estimating dynamic panel data models: a guide for macroeconomists. Economics Letters, 65(1), 9-15. doi: 10.1016/s0165-1765(99)00130-5

[21] Kiviet, J. F. (1995). On bias, inconsistency, and efficiency of various estimators in dynamic panel data models. Journal of Econometrics, 68(1), 53-78. doi: 10.1016/0304-4076(94)01643-e

[22] Lillo, R. L. and Torrecillas, C. (2018). Estimating dynamic Panel data. A practical approach to perform long panels. Revista Colombiana de Estadística, 41(1), 31-52. doi: 10.15446/rce.v41n1.61885

[23] Lokshin, B. (2008). A Monte Carlo comparison of alternative estimators for dynamic panel data models. Applied Economics Letters, 15(1), 15-18. doi: 10.1080/13504850600706545

[24] Mertens, B. (2017). A Comparison of Dynamic Panel Data Estimators Using Monte Carlo Simulations and the Firm Growth Model. Master Thesis. Faculty of Economics and Business Administration, Ghent University.

[25] Soto, M. (2009). System GMM estimation with a small sample. Barcelona Graduate School of Economics Working Paper, 395. https://ideas.repec.org/p/bge/wpaper/395.html 